\title{
Minimal-Invasive Technologies for Treatment of HTS and Keloids: Corticosteroids
}

Juhee Lee and Jihee Kim

\section{Contents}

29.1 Background - 244

29.2 The Corticosteroids - 246

29.3 Pharmacology and Mechanism of Action - 246

29.4 Corticosteroid in Scar Treatment - 247

29.5 Topical Steroids $\mathbf{- 2 4 7}$

29.6 Intralesional Injection - 247

29.6.1 Administration - 247

29.6.2 Side Effects and Complications - 248

29.7 Further Applications - 249

29.7.1 Enhancing the Effect of Intralesional Corticosteroid - 249

29.8 Conclusion - 249

References - 249 


\subsection{Background}

Hypertrophic scars and keloids represent the consequences are the results of abnormal wound healing. Keloids are characterized by overproduction and deposition of collagen and extracellular matrix (ECM). Keloids often manifest as growing and infiltrative lesions toward the neighboring intact skin beyond the original boundaries. Abnormal fibroblast activity is a histopathological hallmark for keloid pathogenesis leading to prominent accumulation of dermal constituents. Due to sustained proliferation without regression, the patients often experience pruritus and pain which leads to functional impairment affecting quality of life. Recent findings have identified that elevated levels of growth factor and cytokines contribute to aberrant fibroblast activity. Among various singling molecules, transforming growth factor beta (TGF- $\beta$ ) family is a crucial mediator in scar formation.

Management of hypertrophic scars and keloids are yet ill categorized. Unlike keloids, hypertrophic scars may resolve partially without treatment, although treatment is required depending on patient desire for further improvement. The use of steroid for the treatment of excessive scarring was first proposed in the 1950s. The early case reports showed that corticosteroids administered during wound healing halted the growth of granulation tissue. In vitro fibroblasts subject to desoxycorticosterone failed to reach maturation, and there were notable morphologic changes [1]. In the following years, small numbers of patients with keloids were treated with systemic intake and regional injection of ACTH who showed inhibition of recurrence. In 1963, triamcinolone was first used for intralesional injection after surgical excision of keloids [1]. Since then, many groups have reported the successful application of intralesionally injected triamcinolone in scar treatment (• Figs. 29.1, 29.2, 29.3, and 29.4).

In the past decades, intralesional injection has been proposed as a standard therapy for hypertrophic scars. The international guidelines on management on keloid scarring have recommended the use of corticosteroids as
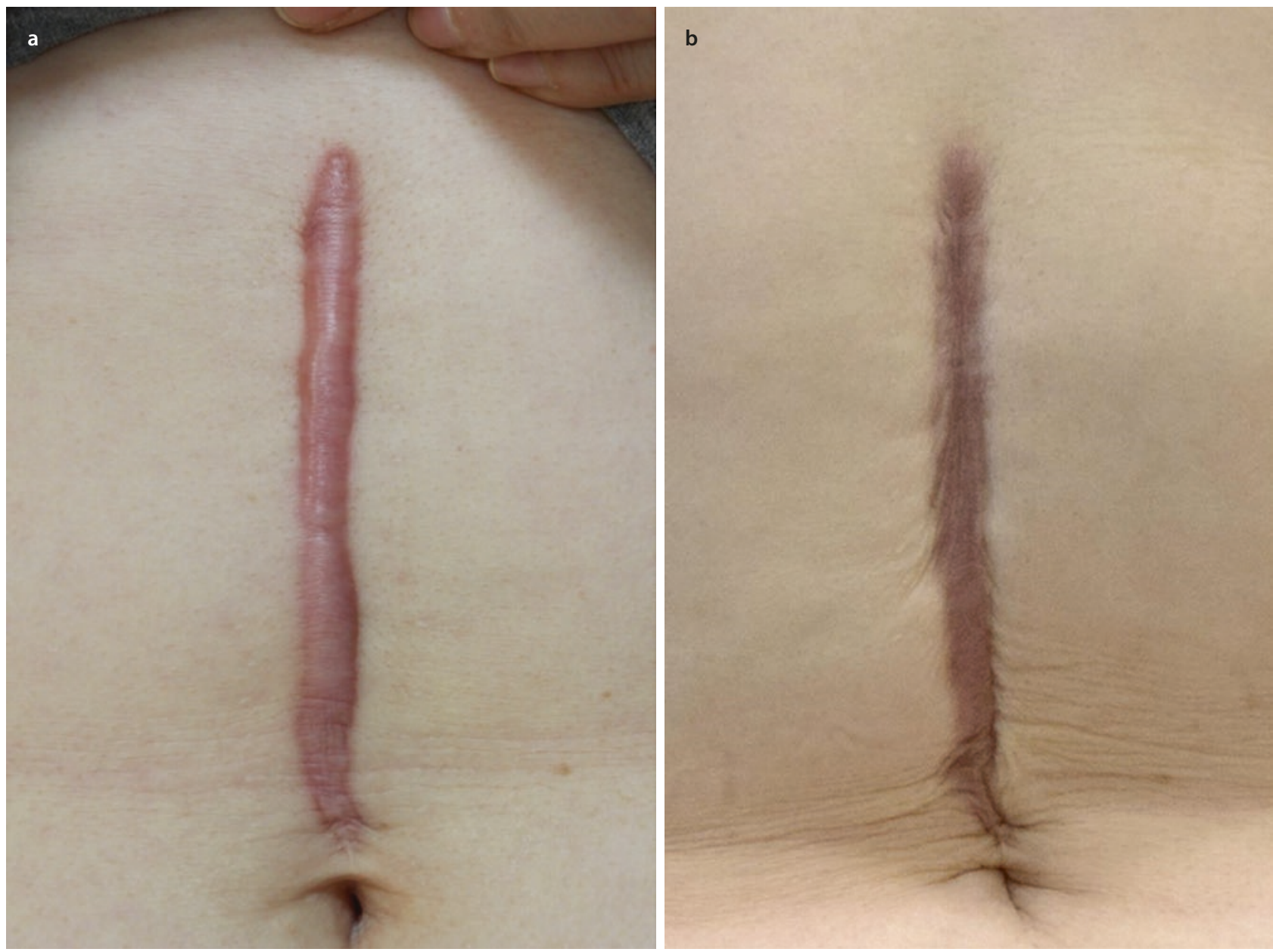

- Fig. 29.1 a A 50-year-old man with keloid after total gastrectomy. b The patient underwent seven sessions of intralesional injection of triamcinolone $(10 \mathrm{mg} / \mathrm{ml})$ in 4 weeks interval 


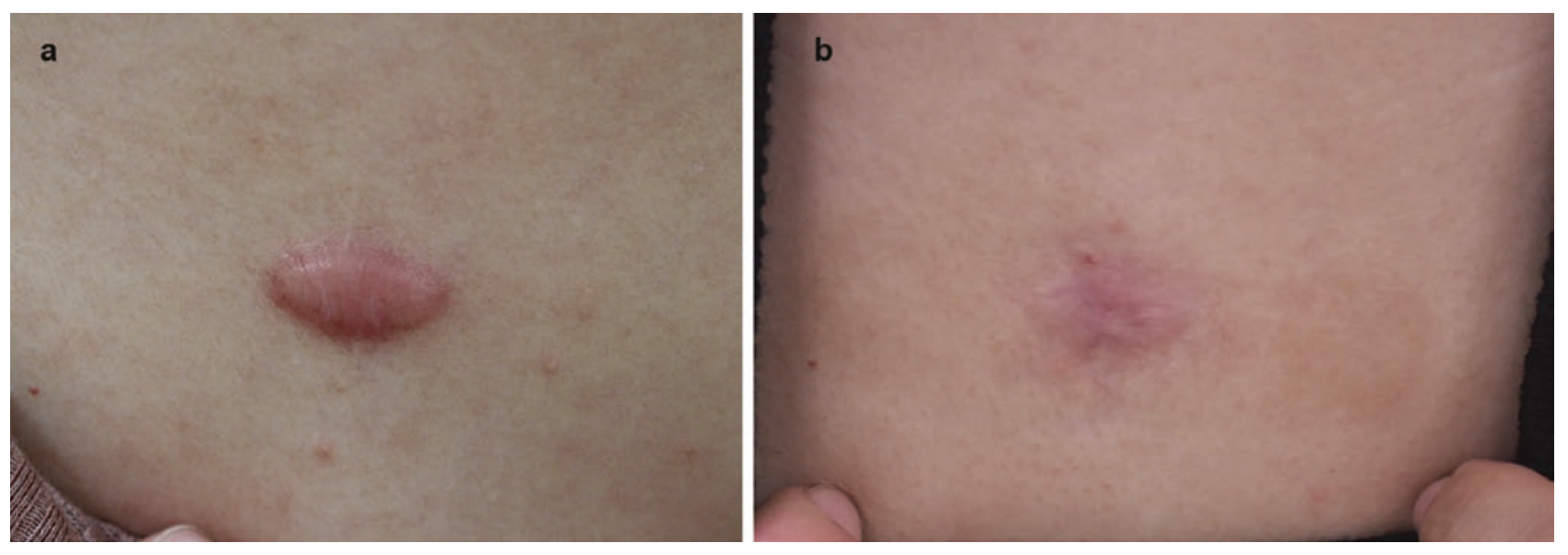

- Fig. 29.2 a A 22-year-old woman with keloid on the chest. b The patient underwent four sessions of fractional $\mathrm{CO}_{2}$ laser $\left(\mathrm{eCO}_{2} \mathrm{Lutronic}\right.$ Goyang, Korea. $60 \mathrm{~mJ}, 15 \%$ density) combined with intralesional injection of triamcinolone acetonide suspension of $10 \mathrm{mg} / \mathrm{ml}$
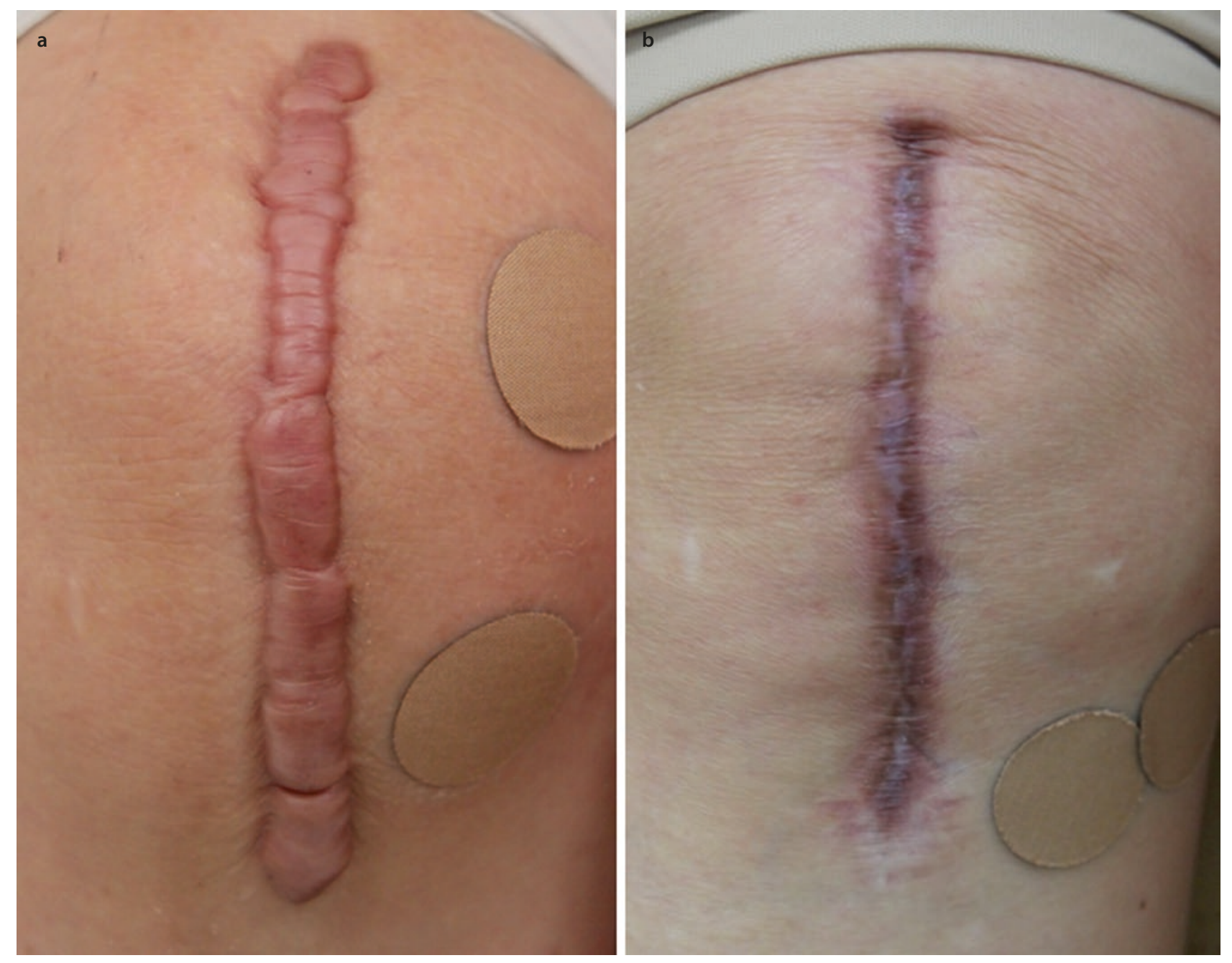

- Fig. 29.3 a A 62-year-old women with keloid after knee replacement surgery. b The patient underwent seven sessions of combination cryotherapy and intralesional injection of triamcinolone $(10 \sim 20 \mathrm{mg} / \mathrm{ml})$ in 4 weeks interval 

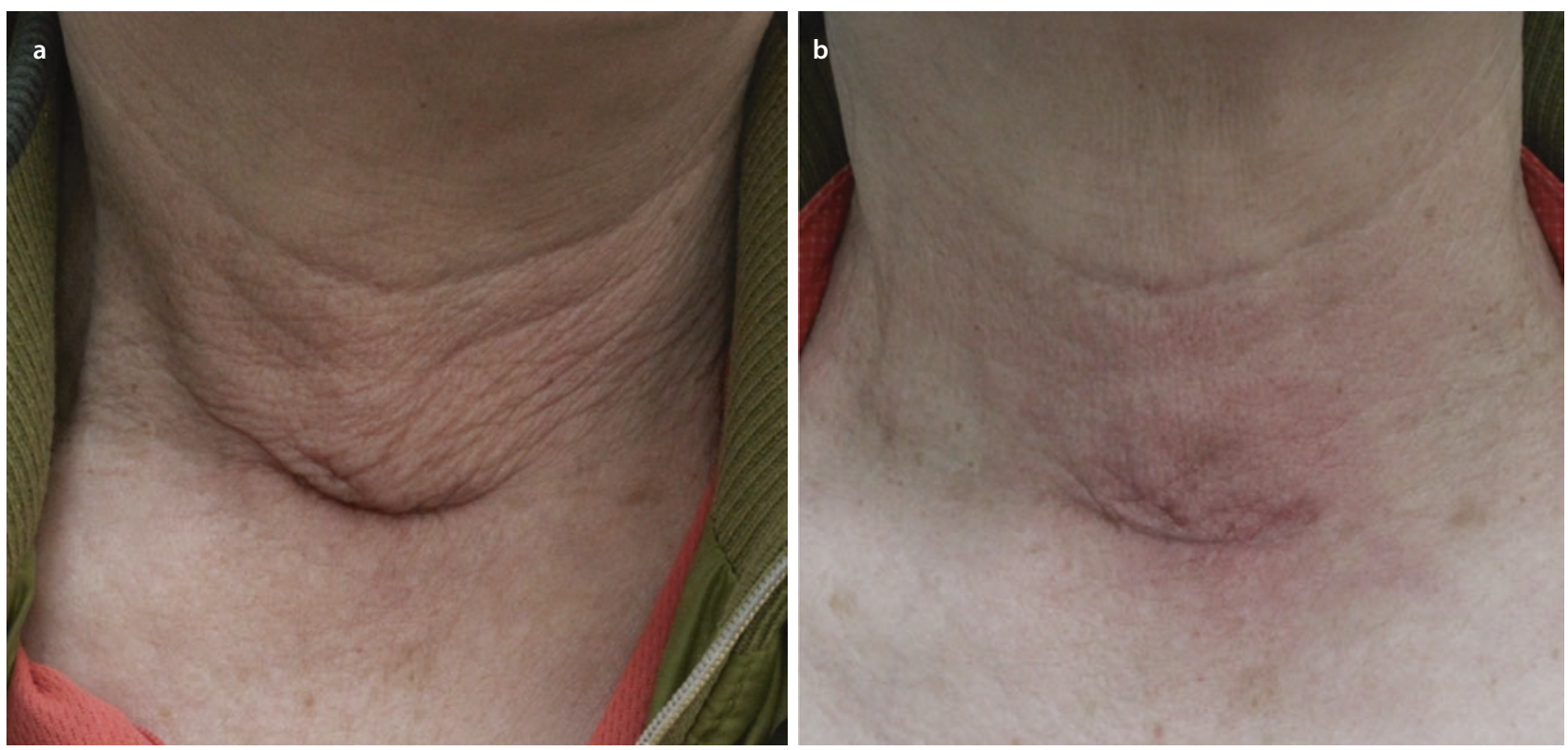

- Fig. 29.4 a A 60-year-old woman visited 12 weeks after total thyroidectomy. b Postoperative lymphedema significantly resolved after single session of intralesional injection of triamcinolone acetonide $(10 \mathrm{mg} / \mathrm{ml})$

a mainstay treatment [2]. Most commonly used corticosteroids for scar treatments are medium- to long-acting agents such as dexamethasone, methylprednisolone, and triamcinolone acetonide. Overall, corticosteroids inhibit all aspects of scar development including inflammation, fibroblast activation, and extracellular matrix accumulations. Thus, the use of corticosteroids efficiently reduces volume of scar tissue and relieves symptoms such as pain and pruritus.

\subsection{The Corticosteroids}

Corticosteroids are the most commonly prescribed anti-inflammatory agent in the medical field. Specific and nonspecific effects of steroids include immunosuppressive, antiproliferative, and vasoconstrictive effects. Besides systemic administration, proper use of topical and intralesional injection requires awareness of its potencies and various formulations.

\subsection{Pharmacology and Mechanism of Action}

All corticosteroids have basic skeletal structure comprising carbon atoms with three hexane rings and one pentane ring. Modifications in the basic cortisol structures result in systemic agents with different potencies, duration of action, metabolism, and mineralocorticoid effects. For example, triamcinolone was synthesized by fluoridation of the hydrocortisone molecule at the 9th position to enhance anti-inflammatory properties.

The free fraction of the corticosteroid enters the cell and exerts its effects by binding to a cytoplasmic glucocorticoid receptor. The glucocorticoid receptor is located within the multi-protein complex consists of heat shock protein and immunophilins. Binding of corticosteroid to its receptor leads to translocation into the nucleus and release from the multiprotein complex. Within the nucleus, the receptor forms a dimer and directly binds to glucocorticoid response elements in the promoter regions of the target genes. Eventually, the intranuclear binding affects the rate of transcription which induces or represses specific target mRNA and protein synthesis. Corticosteroid receptor also interacts with other crucial transcription factors regulating cell metabolism such as cAMP response element binding (CREB) protein. Cellular inflammatory response is modulated via nuclear factor- $\kappa \mathrm{B}(\mathrm{NF}-\kappa \mathrm{B})$. NF- $\mathrm{NB}$ is an important transcription factor that induces the expression of genes regulating inflammatory mediators. Corticosteroids and its receptor inhibit the activity of NF- $\mathrm{KB}$ causing reduction of inflammatory process in cell. Additionally, the glucocorticoid receptor has an inhibitory effect on activator protein 1 (AP-1), which controls the expression of various growth factors and cytokine genes. Other key cytokines or proinflammatory molecule inhibited by glucocorticoids includes tumor necrosis factor- $\alpha$ (TNF$\alpha)$; granulocyte-macrophage colony-stimulating factor (GMCSF); interleukins (IL). IL-1, IL-2, IL-6, IL-8; leukotrienes; and prostaglandins. 


\subsection{Corticosteroid in Scar Treatment}

Unlike normal fibroblasts, keloid fibroblasts possess tumor-like properties, showing excessive proliferation and invasion of surrounding tissues. Enhanced migration and invasion of normal fibroblasts are critical factors for the development of keloids. TGF- $\beta$ signaling has long been considered a pivotal fibrogenic factor in abnormal wound healing. Subsequently, anti-fibrotic strategies based on the blockade or elimination of TGF- $\beta$ signaling emerged as an important pharmacological target for treating keloids [3]. The clinical response to corticosteroids in scar treatments is mainly achieved by reducing the prolonged inflammatory response during wound healing, inhibiting collagen and reducing excessive ECM synthesis by reducing fibroblast activity. Additionally, intralesional injection of corticosteroids enhances fibroblast and collagen degeneration.

In lesions treated with corticosteroids, thick collagen bundles are dissociated and ECM constituents are markedly reduced. In vivo studies have shown that corticosteroid, especially triamcinolone, can retard synthesis of pro-collagen and TGF- $\beta 1$ and TGF- $\beta 2$ expression [4]. Administration of dexamethasone induced reduction in vascular endothelial growth factor and angiogenesis in keloid-derived fibroblasts [5]. Further study on the effect of corticosteroids on keloid pathophysiology is required in the aspect of modulation of chemotaxis immunomodulation controlling fibroblast activity and collagen metabolism.

\subsection{Topical Steroids}

Topical steroids are the most frequently prescribed of all dermatologic drugs, yet its usage has been limited in scar treatment. There are different formulations available in a wide range of potencies and a variety of vehicles. Topical corticosteroids are categorized into four major potency groups and seven classes. The classes are developed based on vasoconstrictor assays and clinical studies which range from class 1 ultra-high potency to class 7 very low potency. Certain formulation is more potent such as ointment formulation, which can enhance percutaneous absorption through increased hydration of the stratum corneum. In selecting a topical glucocorticoid preparation for scar treatment, optimal potency should be selected based on scar extent, location, and thickness. Target site for topical steroids is the viable epidermis or dermis, and the clinical response to a formulation is directly proportional to the concentration of the drug achieved at the target site. When topical application is considered, it is important to monitor potential adverse skin reaction due to continuous application. When skin atrophy or purpura is noted, temporary discontinuation of a topical agent should be considered. For hypertrophic scar or keloid treatment, topical corticosteroids alone have failed to reduce pre-existing scar tissue or prevent scar development and not advocated as a preferred modality.

\subsection{Intralesional Injection}

Keloids and hypertrophic scars are often treated by intralesional injection of therapeutic drugs because the pathogenic target is accumulated collagen and ECM constituents within the dermis. Intralesional administration of corticosteroids allows bypassing the thick stratum corneum barrier and directly treating pathologic dermal lesions with higher concentration of corticosteroids at the site. Triamcinolone acetonide and triamcinolone diacetate are the most widely used corticosteroids for intralesional administration. After its administration, micronized crystals of corticosteroids persist in the skin and released over a period of weeks, thus being the most desirable delivery system for the treatment of chronic inflammatory skin diseases. Triamcinolone agents are available as micronized suspensions of corticosteroid crystals, favored than dexamethasone or betamethasone.

\subsubsection{Administration}

Typically 27 - or 30-gauge needles are the most preferred because it causes less discomfort when penetrating the skin and allow greater precision in injecting the desired quantity. Needles with thicker caliber can be applied for the long-standing lesions with dense tissue. The common therapeutic dosage would be between 10 and $40 \mathrm{mg}$ per $\mathrm{mL}$. Available triamcinolone suspension should be diluted to achieve the final concentration just sufficient to treat the target lesion. Because the injections are administered monthly, the authors favor lower dosage 10 or $20 \mathrm{mg}$ to prevent undesirable effects.

To reduce the discomfort on pain, $1 \%$ or $2 \%$ lidocaine can be used to dilute triamcinolone in desired concentrations. Lidocaine alone may not induce reduction in pain due to its acidity and sodium bicarbonate can be added. Injection with lidocaine and bupivacaine mixture subcutaneously beneath and around the target lesion few minutes prior to the treatment can be beneficial to minimize pain on administration and after the treatment. Other measures to reduce patient discomfort are to apply ice pack or spray cooling system prior to the injection. Topical lidocaine available in tape or cream formulation can be of benefit.

Before administration, it is important to gently shake to suspend the micronized suspensions evenly. Upon injection, the needle should be introduced to target the 

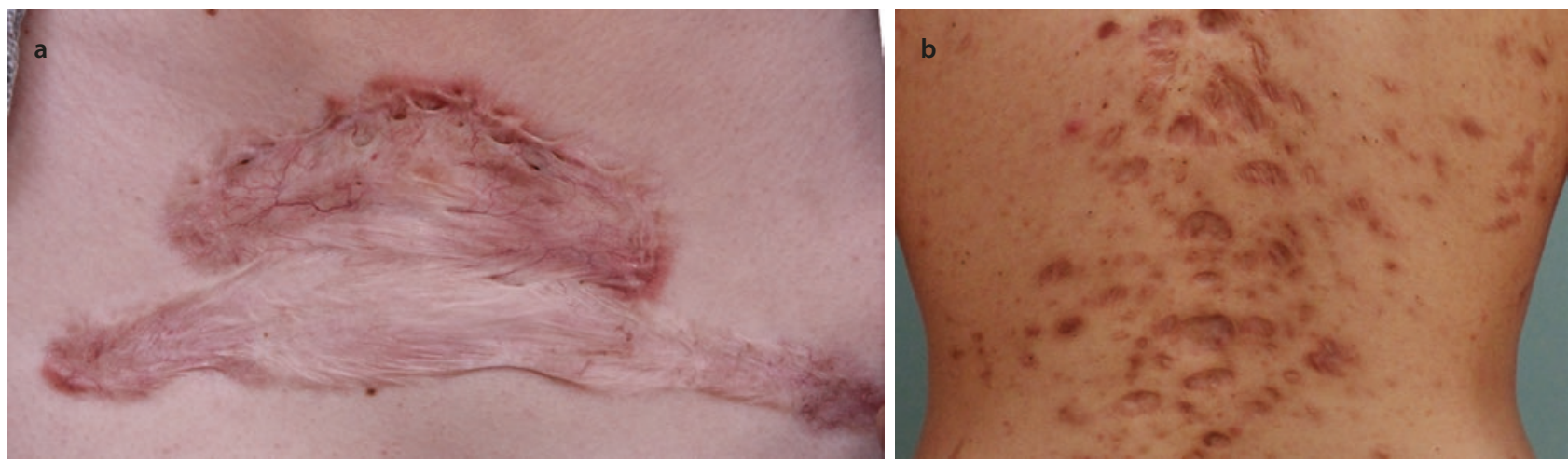

- Fig. 29.5 Localized side effect after corticosteroid intralesional injection. a Telangiectasia on the chest. b Permanent skin atrophy on the back

dermis where the target tissue is deposited. Resistance is felt when correctly injected to the dermis and when the drug is administered in the upper dermis, slight blanching can be noted. Direct injection to the subcutaneous tissue should be avoided which easily induces lipoatrophy. Long-standing keloid tissue with firm texture should be pretreated with liquid nitrogen to induce edema and subsequent softening of the tissue. Alternatively, injection can be performed to the periphery of the scar tissue directing the needle toward the scar tissue. Most treatment algorithms require multiple serial injections of intralesional steroid. In clinical practice, keloids are treated by a monthly injection until the resolution of clinical symptoms. The clinical response may differ between hypertrophic scars and keloids. While hypertrophic scars tend to be more responsive than keloids and generally flatten with time, keloids may require repeated interventions [6].

Currently, intralesional triamcinolone is the major first-line therapy for treatment of both hypertrophic scars and keloids. Its efficacy is well confirmed by numerous clinical trials and meta-analysis [7]. Intralesional triamcinolone injection effectively induced marked reduction in the size of the keloid. It was particularly efficient when administered after surgical removal of keloid lesions. Intralesional triamcinolone immediately after wound closure and at early postoperative visits, $20 \mathrm{mg} / \mathrm{ml}$ and $10 \mathrm{mg} / \mathrm{ml}$ respectively, resulted in $76.5 \%$ recurrence-free resolutions during a follow-up period of 18 months [8]. Combination treatment regimen with intralesional injection, $20 \sim 40 \mathrm{mg} / \mathrm{ml}$, and potent topical steroid application yield favorable results with $85.7 \%$ recurrence-free on average of 32-month followup period [9]. Given the results of the clinical studies encompass different lesions occurring on different anatomical locations and durations, further clinical trials should be directed to overcome limitations of current repots including differences in timing of intervention, anatomical locations, inclusion and exclusion criteria, and inconsistent outcome measures.

\subsubsection{Side Effects and Complications}

Steroid-induced side effects include skin atrophy, telangiectasia, hypopigmentation, ulceration, and, rarely, systemic complications. Fortunately, the most commonly encountered side effects of intralesional corticosteroid injection are localized. The incidence of localized side effects is often reported in one-third of patients. Skin atrophy usually occurs over several weeks to months after the injection and may resolve spontaneously without treatment. However, atrophic lesion caused by repeated injection with high dosage persists longer and may induce permanent change. Telangiectasia may occur frequently on the site of injection. In most cases, the lesions do not regress spontaneously and further treatment is required. Laser or light devices such as pulsed dye laser or intensive pulsed light can be successfully applied for its treatment. Foreign body reaction to intralesional triamcinolone is rare but reported. Granulomatous reactions result from the failure of injected crystalized corticosteroid particle to disperse or incomplete absorption. Clinically, prolonged absorption of injected material presents as xanthoma-like lesions and resolves spontaneously over few months (• Fig. 29.5).

Systemic complications are rare but occasionally observed. In children, cases of Cushing's syndrome following repeated injection of high dosage intralesional steroid is reported. In adults, the evidence for systemic complication is limited with few reported cases of Cushing's syndrome when patients are subjected to large doses due to extensive keloids. Monthly injection of large dosage of corticosteroids is often brought to account to cause menstrual irregularities. Although there is insufficient clinical evidence, it is unlikely that intralesional corticosteroid alone can cause adrenal complications.

Complications related to intralesional injections can be prevented by using the lowest concentration and the 
smallest quantity of the drug needed. Increasing the concentration can be adjusted as needed after the repeated course of treatments. For patients with multiple or large lesions, alternating the treatment site or dilution to lower dosage is recommended. Additionally, scars across the joint area and periorbital area can be more susceptible to localized absorption of corticosteroids and higher concentration should be avoided. Although diabetes is not a contraindication to intralesional corticosteroid injection, patients should be approached with caution and appropriate monitoring should be considered.

\subsection{Further Applications}

\subsubsection{Enhancing the Effect of Intralesional Corticosteroid}

For long-standing hypertrophic scar lesions or large and firm keloids, the combination with cryotherapy can be highly effective. Cryotherapy with liquid nitrogen induces dissociation of accumulated ECM tissue along with possible vascular suppression and apoptosis of fibroblasts. Open spray technique is most commonly used in combination with intralesional injections. When used in combination, injections should be made after defrosting of the lesion. There are several reports showing marked improvement of hypertrophic scars and keloids after either superficial or intralesional cryotherapy. To prevent hypopigmentation from melanocyte destruction or other associated side effects of cryotherapy, shorter exposure (10 30 seconds) is recommended with fewer (two to three) freeze thaw cycles.

In addition to intralesional injection of triamcinolone, localized injection of antimetabolite agents are reported to be beneficial for scar treatments. 5-Fluorouracil (5-FU) is an antimetabolite inhibiting DNA synthesis which increases fibroblast apoptosis and inhibit proliferation. The use of combination triamcinolone and 5-FU has been demonstrated to be as efficacious as triamcinolone alone. Although there are possible side effects from 5 -FU including injection site irritation or delayed wound healing, it lacks atrophy or erythema associated with corticosteroid injections. The pain upon administration was a major drawback in 5-FU treatment, while its combination with triamcinolone relieved excruciating pain. Combination of intralesional 5-FU injection with topical corticosteroids yields successful result in scar treatment [3]. Given the requirement for repeated treatment sessions and pain associated with intralesional injection of corticosteroids, other modalities such as lasers and efficient delivery systems are suggested.

\subsection{Conclusion}

The management of keloids and hypertrophic scars continues to challenge clinicians, and there is no universally accepted treatment algorithm. Although there are myriad of treatment options, selection of treatment modality largely depends on the patient desire. The use of corticosteroids has remained mainstay of the treatment for more than half century. While potent topical corticosteroids can be of limited use to address pruritus, intralesional injection of triamcinolone is the mainstay in treatment. Intralesional triamcinolone injection is thought to reduce scar formation by suppressing inflammation, inhibiting fibroblast proliferation, and inducing collagen remodeling. Corticosteroid is cost-effective because it does not require additional equipment, and thus readily applied in patients' regular clinical visits. Further studies are warranted to identify the optimal combination with other modalities for scar treatment.

\footnotetext{
- Take-Home Messages

- Intralesional injection of corticosteroid is effective first-line treatment for the treatment for keloids and hypertrophic scars.

- Corticosteroid inhibits fibroblast growth proliferation and collagen synthesis by effecting on TGF- $\beta$ signaling and promoting collagen degeneration.

- Intralesional injection of triamcinolone acetonide $(10-40 \mathrm{mg} / \mathrm{ml})$ is desired and lower concentration is more favorable for the initial treatments.

- The use of smaller gauge needle and syringe allows better control of the injection and minimize patient discomfort.

- For long-standing and firm scar lesions, combination with cryotherapy is required to achieve optimal penetration.
}

\section{References}

1. Ketchum LD, Smith J, Robinson DW, Masters FW. The treatment of hypertrophic scar, keloid and scar contracture by triamcinolone acetonide. Plast Reconstr Surg. 1966;38(3):209-18.

2. Gold MH, Berman B, Clementoni MT, Gauglitz GG, Nahai F, Murcia C. Updated international clinical recommendations on scar management: part 1-evaluating the evidence. Dermatol Surg. 2014;40(8):817-24. https://doi.org/10.1111/ dsu.0000000000000049.

3. Tziotzios C, Profyris C, Sterling J. Cutaneous scarring: pathophysiology, molecular mechanisms, and scar reduction therapeutics Part II. Strategies to reduce scar formation after dermatologic procedures. J Am Acad Dermatol. 2012;66(1):13-24. https://doi. org/10.1016/j.jaad.2011.08.035. 
4. Rutkowski D, Syed F, Matthews LC, Ray DW, McGrouther DA, Watson REB, et al. An abnormality in glucocorticoid receptor expression differentiates steroid responders from nonresponders in keloid disease. Br J Dermatol. 2015;173(3):690-700. https:// doi.org/10.1111/bjd.13752.

5. Wu WS, Wang FS, Yang KD, Huang CC, Kuo YR. Dexamethasone induction of keloid regression through effective suppression of VEGF expression and keloid fibroblast proliferation. J Invest Dermatol. 2006;126(6):1264-71. https://doi.org/10.1038/ sj.jid. 5700274 .

6. Kim S, Choi TH, Liu W, Ogawa R, Suh JS, Mustoe TA. Update on scar management: guidelines for treating Asian patients. Plast Reconstr Surg. 2013;132(6):1580-9. https://doi.org/10.1097/ PRS.0b013e3182a8070c.
7. Wong TS, Li JZ, Chen S, Chan JY, Gao W. The efficacy of triamcinolone acetonide in keloid treatment: a systematic review and meta-analysis. Front Med (Lausanne). 2016;3:71. https:// doi.org/10.3389/fmed.2016.00071.

8. Hayashi T, Furukawa H, Oyama A, Funayama E, Saito A, Murao N, et al. A new uniform protocol of combined corticosteroid injections and ointment application reduces recurrence rates after surgical keloid/hypertrophic scar excision. Dermatol Surg. 2012;38(6):893-7. https://doi.org/10.1111/j.15244725.2012.02345.x.

9. Ledon JA, Savas J, Franca K, Chacon A, Nouri K. Intralesional treatment for keloids and hypertrophic scars: a review. Dermatol Surg. 2013;39(12):1745-57. https://doi.org/10.1111/dsu.12346.

Open Access This chapter is licensed under the terms of the Creative Commons Attribution 4.0 International License (http://creativecommons. org/licenses/by/4.0/), which permits use, sharing, adaptation, distribution and reproduction in any medium or format, as long as you give appropriate credit to the original author(s) and the source, provide a link to the Creative Commons license and indicate if changes were made.

The images or other third party material in this chapter are included in the chapter's Creative Commons license, unless indicated otherwise in a credit line to the material. If material is not included in the chapter's Creative Commons license and your intended use is not permitted by statutory regulation or exceeds the permitted use, you will need to obtain permission directly from the copyright holder. 